\title{
Enhanced Teaching and Learning in Media Richness and Media Synchronicity Environments
}

\author{
Newman John H \\ School of Business Coppin State University2500 W. North Avenue Baltimore, MD 21216 USA \\ *Corresponding Author: jnewman@coppin.edu
}

Copyright (C) 2014 Horizon Research Publishing All rights reserved.

\begin{abstract}
This paper seeks to investigate the selection of various communication media as they correspond to the subject matter and learning models. It examines relationships between and among the properties of the media used for communication and the underlying learning model used in instruction. The intended result is to enable a more informed selection of educational environments.
\end{abstract}

Keywords Technology and Learning, Instructional Design, Management Information Systems, Information Technology, Media Richness Theory, Media Synchronicity Theory, Pedagogical Evolution, Revision of Technology, Web-based Learning

\section{Introduction}

The ongoing advances in information technology offer diverse opportunities to develop learning environments that transcend the traditional same time/same place model. However, the selection among the various environments must be compatible with the subject matter conveyed in order to have the best potential for positive outcomes.

Online learning or distance education incorporates separation of instructor and student, use of communication media, and two-way communication between instructor and student Bates [2]. Within an online learning environment, the instructor and student are separated in space. This absence of a fixed meeting place allows flexibility in educational activities. If this spatial separation is accompanied by temporal separation the process is called asynchronous distance education. The limitations inherent in the separation of student and instructor are addressed by the selection and use of communication media. If this is two-way communication, the student is part of an organized educational process; if not, the student is pursuing a form of self-improvement. The selection of learning environment and communication media should not be made without a thorough study of the subject matter intended to be conveyed. In addition, computer competency has become as necessary as writing competency and it is necessary for educational institutions to adopt computer literacy requirements for their students. Unlike Web-based courses such as Information Systems, where the medium is the message, the first question to ask is whether the context determines the nature of the knowledge to be learned. This is an important question because different sets of contextual practice related to the knowledge in question need to be acquired in order for learning to be successful Arsham, [1].

Two established theories addressing these issues are Media Richness Theory (MRT) and Media Synchronicity Theory (MST).Both will be examined and conclusions will be offered regarding the role of the subject matter in the selection of learning environment and communication media.

\section{Media Richness Theory}

Media Richness Theory (MRT) was developed to explain how managers/instructors choose communications media Daft et al.

MRT proposes that information is conveyed to reduce uncertainty and reduce equivocality. Uncertainty is the result of the absence of complete information; equivocality is the result of multiple interpretations of the same information.

The criteria for classifying media "richness" (information-carrying capacity) include the availability of immediate feedback, the number of channels utilized, personalization, and language variety Daftet al, [5].

MRT suggests that media of lower richness are more effective when the purpose is to reduce uncertainty while media of higher richness are more effective when the purpose is to minimize equivocal interpretations of the information conveyed, i. e., to reduce equivocality.

Face to face (F2F) offers immediate feedback and can use multiple channels of communication (verbal, body language, facial expressions). It is the richest medium. Other communications media range in richness along a continuum from $(\mathrm{F} 2 \mathrm{~F})$ to the staid numerical reports. There is empirical support for matching communications media with subject matter that has been analyzed in order to prescribe a media fit Donnabedian et al, [7]. 
Distance education can mimic this continuum. A canned cartridge can be incorporated into any of the commercial educational software programs. Subjects in the hard sciences like mathematics can then be presented to users. At the other extreme, extensive use of virtual classrooms, discussion boards, synchronous online sessions, etc., can approach the F2F model and better present seminars and programs in the arts (Bates, 1995).This is not to suggest that distance education practices cannot be adopted into the F2F classroom. Indeed, this practice may be seriously underutilized (Scagnoli, 2005).

\section{Media Synchronicity Theory}

Media Synchronicity Theory (MST) is a second established theory. Under MST, communication has two fundamental purposes, conveyance and convergence.

Conveyance is the distribution of as much relevant information as possible to aid understanding.

Convergence seeks understanding each individual's interpretation of information - not the information itself. This MST dichotomy of purpose is consistent with the two major classes learning models that seek to describe and predict how learning occurs: objectivism and constructivism.

Objectivism argues that the goal of learning is to understand the objective reality and act accordingly. This class of learning models implies that the goal of communicating is to effectively and efficiently transmit unambiguous knowledge from the instructor to the student (Leider et al [8].This model has been incorporated into the Learning Object approach that “... offers promises of universal access to reusable online materials" (Nurmi\&Jaakkola, 2005).

Constructivism holds that the mind should not reproduce an external reality but rather produce its own conception of events. Students analyze different interpretations of information, form abstract concepts to represent reality, and actively participate in their learning process. The communicator is really a mediator (Schreiber \& Berge, 1998).

MST suggests that there are five media characteristics that can affect communication. These characteristics are: immediacy of feedback, symbol variety, parallelism, rehearsability, and reprocessability Dennis et al [6].These five concepts are defined below.

1. Immediacy of feedback is the degree to which the medium supports rapid response to the communications received. 2. Symbol variety is the way or ways through which the medium can communicate information. 3 . Parallelism is the ability of the medium to support multiple conversations simultaneously. 4. Rehearsability is the extent to which the medium enables the senders to fine-tune their messages before sending them. 5. Reprocessability is the extent to which a message can be reexamined within the context of the communication event.

Analysis of purpose of the communication is essential to the proper application of MST. If the purpose is conveyance, media should be chosen that exhibit low immediacy of feedback and high reprocessability. This enables delivering the maximum amount of information with the minimum amount of feedback while allowing the information to be reexamined within the communication event.

If the purpose of the communication is convergence, media should be chosen that have high immediacy of feedback and high parallelism. Immediate feedback and support for multiple simultaneous conversations can lead to a shared interpretation of the information communicated - the goal of convergence. These characteristics are summarized in Table 1.

Table 1. Methodology Choice Based Upon Purpose of Communication

\begin{tabular}{|c|c|}
\hline CONVEYANCE & CONVERGENCE \\
\hline Feedbacklow & Feedbackhigh \\
\hline Symbol Varietylow & Symbol Varietyhigh \\
\hline Parallelismhigh & Parallelismlow \\
\hline Rehearsabilitylow & Rehearsabilityhigh \\
\hline Reprocessabilityhigh & Reprocessabilityhigh \\
\hline
\end{tabular}

\section{Empirical Testing of MRT and MST}

Empirical support for MRT has yielded inconsistent results on whether the theory satisfactorily explains why the choice of a particular communications media was made. There has been more consistency in evaluating MRT as a prescriptive theory - how to make the choice of media based upon performance measures from when that media was used in the past (Dennis et al [6]; Donabedian, et al [7].

There is empirical support for MST proposition that media of low feedback and high parallelism are better for conveyance processes while media of high feedback and low parallelism are better for convergence processes. There is only qualified support for the other MST propositions (Carswell, [4].

\section{Policy Framework}

The selection and use of communications media for online educational technologies must be made with an awareness that these media are in flux. Specialized course authoring packages including Blackboard and WebCT are in competition with each other and with packages developed in-house by colleges and universities. All must also contend with newsgroups, Web pages, email distribution lists, and the like (Brent, [3].

In addition, online courses must be revised unlike F2F courses that evolve naturally. While instructors in F2F courses add things that work and discard thing that don't, instructors in online courses deal with a main body of established material. It is necessary to revise the ways in which that material is used to accommodate different times, 
different students, and different circumstances in the world at large surrounding the material (Ong, [9]; Goldhaber, [7].

\section{Experiment}

Two upper level undergraduate classes were taught in the fall, 2013 term of a small, urban university in the northeastern United States. A project management class was taught with high emphasis on media richness. The same professor also taught an operations research (OR) class with high emphasis on media synchronicity. The project management class (pman) was a hands-on planning and implementation of a real project - current student to potential student recruitment. The operations research class was taught by giving students all relevant information available and then seeking individual interpretations regarding the solutions to problems or the maximization of opportunities. The two classes were taught by the same professor to students from the same student body. The subject matter of the classes was similar. Both were taught F2F. Two measures were used: the professor's grading of the students and the students' grading of the professor. The pman class was straight A's with one exception. The OR class was close to the standard distribution with the class average of $\mathrm{C}+$. The pman class rated the professor 4.99 out of 5 while the OR class rated the professor 4.15 out of 5 .

\section{Conclusions}

Advances in technology have led to more effective and efficient communications media. These media can be used in distance education. However, one size does not fit all. The selection and use of communications media should be related to the subject matter intended to be conveyed and the underlying learning model used ininstruction.

The above experiment strongly supported media richness and heavy student involvement. However, it can be said that the media synchronicity methodology conveyed more relevant information and thus required more student interpretation.

The lasting conclusion is one of awareness. Any selection and use of media must be made with the awareness that the media selected are in flux and the material conveyed is subject to ongoing revision.

\section{REFERENCES}

[1] Arsham, H. (2002). Impact of the Internet on learning and teaching. USDLA Journal, Vol. 16: No. 3

[2] Bates, A.W. (1995). Technology, open learning, and distance education. London and New York: Routledge.

[3] Brent, D. (2005). Teaching as performance in the electronic classroom. First Monday, volume 10, number 4 (April, 2005).

[4] Carswell, A. (2001). Facilitating Student Learning in an Asynchronous Learning Network. Unpublished Dissertation, University of Maryland.

[5] Daft, R., \& Lengel, R. (1986). Organizational information requirements, media richness, and structural design. Management science, 32(5), 554-571.

[6] Dennis, A.R., \& Valacich, J.S. (1999). Rethinking media richness: Towards a theory of media synchronicity. Paper presented at the HICSS, Hawaii.

[7] Donabedian, B., S. M. McKinnon, and W. J. Bruns Jr. "Task Characteristics, Managerial Socialization, and Media Selection." Management Communication Quarterly 11 (February 1998): 372-400.

[8] Goldhaber, M. H. (2004). The mentality of homo interneticus: some ongian postulates. First Monday, volume 9, number 6 (June, 2004).

[9] Leidner, D.E., \& Jarvenpaa, S. L. (1995). The use of information technology to enhance management school education. MIS quarterly, 19(3, 265.

[10] Nurmi, S. \&Jaakkola, T. (2005) Problems underlying the learning object approach.International journal of instructional technology.

[11] Ong, W. (1982). Orality and literacy: the technologizing of the world. New York: Methuen.

[12] Scagnoli, N. (2005). Impact of online education on traditional campus-based education. ITDL Journal, http://itdl.org/Journal/Oct_05/article06.htm

[13] Schreiber, D., \& Berge, Z. (Eds.). (1998) Distance training. San Francisco: Jossey-Bass Publishers. 